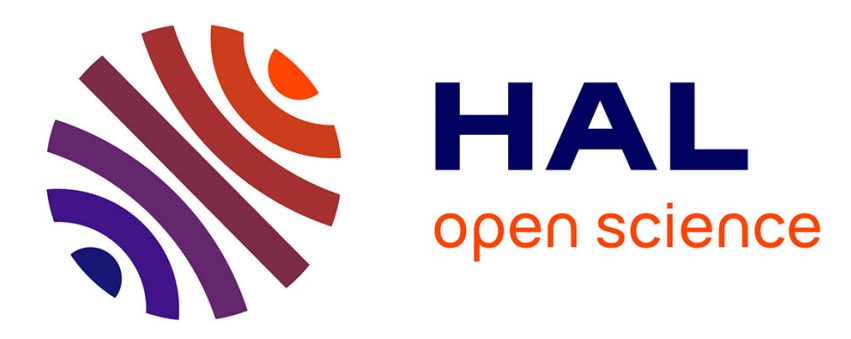

\title{
Intermittency in a Locally Forced Plane Couette Flow
}

S. Bottin, Olivier Dauchot, F. Daviaud

\section{To cite this version:}

S. Bottin, Olivier Dauchot, F. Daviaud. Intermittency in a Locally Forced Plane Couette Flow. Physical Review Letters, 1997, 79, pp.4377-4380. 10.1103/PhysRevLett.79.4377 . cea-01373959

\section{HAL Id: cea-01373959 https://hal-cea.archives-ouvertes.fr/cea-01373959}

Submitted on 29 Sep 2016

HAL is a multi-disciplinary open access archive for the deposit and dissemination of scientific research documents, whether they are published or not. The documents may come from teaching and research institutions in France or abroad, or from public or private research centers.
L'archive ouverte pluridisciplinaire $\mathbf{H A L}$, est destinée au dépôt et à la diffusion de documents scientifiques de niveau recherche, publiés ou non, émanant des établissements d'enseignement et de recherche français ou étrangers, des laboratoires publics ou privés. 


\title{
Intermittency in a Locally Forced Plane Couette Flow
}

\author{
S. Bottin, O. Dauchot, and F. Daviaud \\ Groupe Instabilités et Turbulence, SPEC, CEA Saclay, F-91191 Gif-sur-Yvette, France
}

(Received 14 April 1997)

\begin{abstract}
The plane Couette flow displays a globally subcritical transition to turbulence. When forced by a thin wire introduced in the central plane, the basic flow bifurcates towards a new state of streamwise vortices which break down through a complex spatiotemporal regime. When a bead is introduced in the central plane, only a few vortices are generated. Their destabilization occurs via a new type of temporal intermittency hard to understand within the framework of dynamical systems. [S0031-9007(97)04572-9]
\end{abstract}

PACS numbers: 47.20.Dr, 47.15. $-\mathrm{x}, 47.20 . \mathrm{Ky}, 47.27 . \mathrm{Cn}$

In spite of impressive progress, the problem of the transition to chaos and turbulence still offers numerous open issues. Both manifest themselves as irregular regimes. Temporal chaos is associated with systems where few degrees of freedom are active owing to confinement effects, for which scenarios have been identified [1]. By contrast, turbulence involves many spatial and temporal scales. In between one finds spatiotemporal chaos where the dynamics of ordered structures remains an important ingredient [2].

In trying to identify steps in the transition process, a first distinction can be made from the nature of the primary bifurcation. Supercritical transitions are characterized by continuous growth of the distance to the basic state in the direction of some known unstable mode. Rayleigh-Bénard convection is a typical example. A scenario then develops, for which classical tools of (weakly) nonlinear analysis are available. Another, strongly different, situation occurs when the primary bifurcation is subcritical. In that case, our understanding is much more limited and entirely relies on our capability to determine branching solutions that become stable at finite distance of the basic state. In the realm of hydrodynamics, the plane Couette flow (PCF) corresponds to this situation since it is known to be linearly stable for all Reynolds numbers [3], whereas transition to turbulence is observed in experiments [4]. The PCF therefore seems to be a good prototype for studying "globally subcritical" transitions to turbulence [5], and has been the subject of numerous studies accordingly.

In order to reach those nonlinear branching solutions the most direct way is to excite the flow by an instantaneous localized perturbation which serves as the initial condition [6-8]. Turbulent spots generated in that way present a core of small scale turbulence surrounded by streaky structures which are also observed when they relax [9]. Another possibility has been explored by considering slight modifications of the original PCF configuration numerically [10]. Experimentally, this has also been done by introducing a thin wire parallel to the spanwise direction by Dauchot and Daviaud [11] who succeeded in stabilizing streamwise counterrotating vortices. Further investigations have con- firmed their role in the transition at the limit of the pure PCF [9]. In this context, we first study the transition scenario involving the breakdown of the streaks along the wire leading to a quasi-1D spatiotemporal regime. Then, in an attempt to understand it, we consider a modified experiment where the localization of perturbations around a bead placed in the middle of the flow yields a temporally intermittent dynamics.

Our PCF setup is made of an endless transparent plastic band guided by rollers achieving a shear flow without mean advection (see [6] for details). The Reynolds number is defined as $R=U h / \nu$, where $U$ is the speed of the wall, $h=3.5 \mathrm{~mm}$ is half of the gap, and $\nu$ is the kinematic viscosity of water. The accuracy on the determination of $R$ is within $3 \%$. Geometrically, we define the streamwise direction as $x$, the normal to the wall direction as $y$, and the spanwise direction as $z$. The test section is about $1 \mathrm{~m}$ long and using $2 h$ as the length scale, the spanwise aspect ratio is $\Gamma_{z} \approx 35$ and the streamwise aspect ratio is $\Gamma_{x} \approx 190$. The flow can be forced in two ways. First, as in [11] a thin wire of radius $\rho / h \in[0.0035,0.1714]$, parallel to the spanwise direction, is introduced in the central plane. Second, as shown in Fig. 1, a little bead of radius $r / h=0.35$ is placed in the central plane and chosen as the origin $(0,0,0)$ of the reference frame. The bead is held by a thin wire parallel to the streamwise direction that does not disturb the flow, as shown in [9]. The $(y, z)$ structure of the flow close to the perturbation is revealed

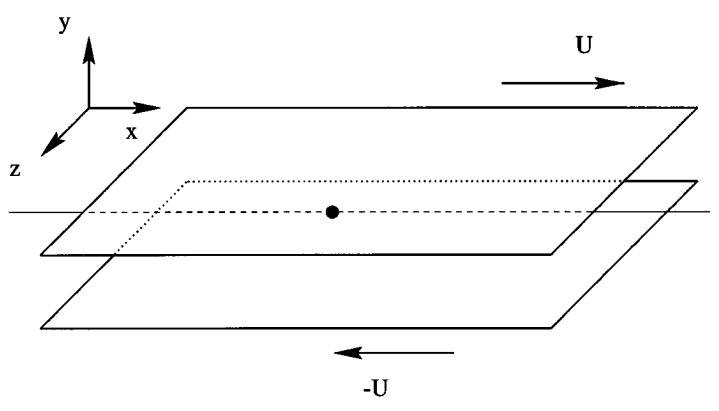

FIG. 1. Modified Couette flow apparatus. 
from the light of a laser sheet diffused by a yellow dye introduced close to the belt, at one entrance of the channel. Its temporal behavior is studied using another laser sheet in the $(x, z)$ plane, illuminating the flow seeded with iriodin.

Figure 2 displays the bifurcation diagram of the flow perturbed by the thin wire when varying $\rho / h$. Upon increasing $R$, the first bifurcation of the basic state, observed at $R=R_{0}$, is towards streamwise vortices extending all around the wire. For sufficiently large $\rho / h$ typically $>0.02$ the following sequence is observed: At a larger $R=R_{1}$, a second bifurcation takes place, where the streamwise vortices are destabilized by localized turbulent bursts. A complex intermittent spatiotemporal regime sets in: A turbulent burst develops at some place, lasts for a while, interacts strongly with the streamwise vortices, and then relaxes. This process repeats itself elsewhere later. Such a regime is usually called spatiotemporal intermittency, at least in a loose sense [12]. For $R>R_{2}$, the turbulent bursts merge to form a sustained band of turbulence all along the wire. Now, decreasing $R$ from above $R_{2}$, the band of turbulence remains sustained down to $R_{3} \simeq R_{2}$. By contrast for smaller $\rho / h$, the transition from streamwise vortices to turbulence is essentially direct and $R_{1} \simeq R_{2}$, while hysteresis is observed for the reverse transition as $R$ is decreased $\left(R_{3} \simeq 325 \pm 5\right)$. A striking feature of this bifurcation diagram is that, throughout the $\rho / h$ range, sustained turbulence can be observed above $R=R_{3} \simeq 325$ which is precisely the value found for the minimal Reynolds number beyond which turbulent spots can be generated in pure PCF [8].

The perturbation introduced by the wire preserves translational invariance in the spanwise direction. As a consequence, a quasi-one-dimensional regime of (provisionally called) spatiotemporal intermittency, is obtained. By contrast, using the bead, i.e., a pointwise perturbation, gives rise to spatially localized processes. Below some

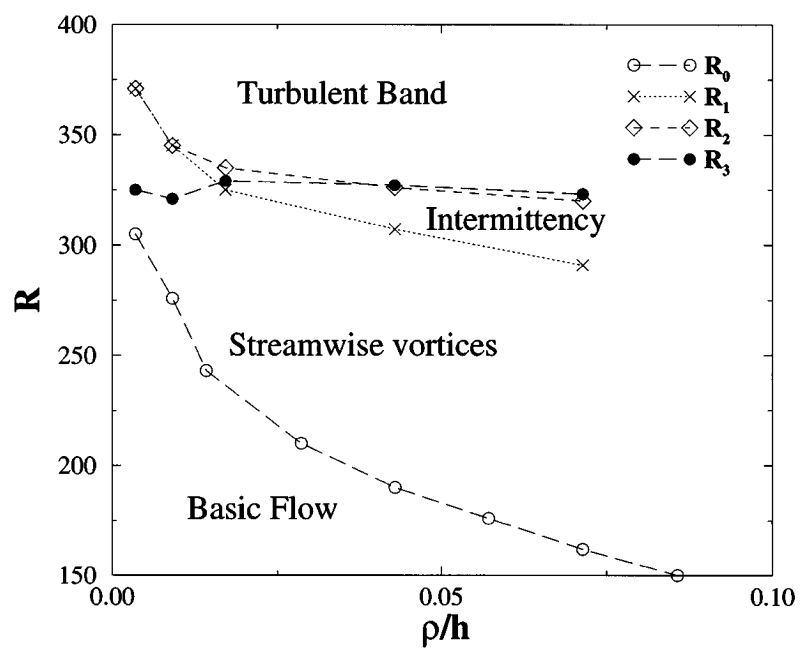

FIG. 2. Evolution of the phase diagram with $\rho / h$ for the wire. threshold $R_{0}^{\prime}$ (=105 \pm 3 in the present experiment with $\rho / h=0.35)$, the flow remains featureless.

For $R>R_{0}^{\prime}$, four pairs of stationary counterrotating streamwise vortices are indeed stabilized: two on each side of the bead. Figure 3(b) displays a $(y, z)$ cut through the two pairs of vortices on the right hand side of the structure in Fig. 3(a). These vortices are similar to those observed along the wire and described in previous studies $[9,11]$ : They occupy the whole gap, their separation is of the same order as the wavelength of the streamwise vortices, and their symmetries are comparable. The pairs of vortices are labeled ( $a, b, c$, and $d$ ) as shown in Fig. 3(a). Pairs $a$ and $b$ are symmetrical with respect to a reflection about the $(x, y)$ plane. Pairs $a$ and $c$ are axially symmetrical with respect to the $z$ axis.

For $R>R_{1}^{\prime}(\simeq 300)$ the vortices become unstable and bursts of turbulence are observed around the bead. However, turbulence is not sustained but intermittent. Preliminary observations indicate complex processes involving vortex merging, creation, and breakdown which are still under study. Turbulent bursts interrupting laminar intermissions are spatially similar to turbulent spots generated by instantaneous perturbations [6-8]. They can develop to become as wide as the span $L_{z}$. Turbulent domains so created then collapse (relaminarization) homogenously all over their surface. A relaxation towards a brief stage of featureless flow is observed. Afterwards the pairs of streamwise vortices, henceforth called the laminar state, soon reappear.

For $R>R_{2}^{\prime}=325$, when a turbulent burst occurs, it no longer decays but turns into a sustained turbulent spot. No hysteresis is observed when decreasing $R\left(R_{3}^{\prime}=R_{2}^{\prime}\right)$.

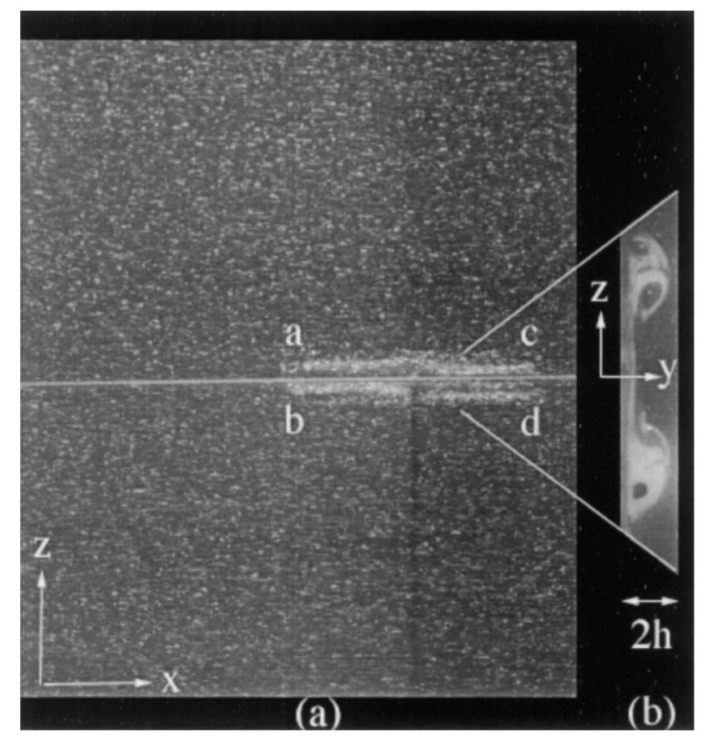

FIG. 3. Photograph of the flow displaying (a) four streaks or pairs of streamwise vortices in the $(x, y)$ plane close to the bead. (b) Section in the $(y, z)$ plane of two pairs of counterrotating streamwise vortices. 
The global phenomenon is very robust and does not seem to be sensitive to boundary effects. Several experiments have been performed with different layouts, varying the bead diameter, its position, and the noise level. Whereas the thresholds were slightly dependent on the experimental conditions, the intermittent behavior could be observed on roughly the same range of Reynolds numbers.

Quantitatively, the intermittent regime has been characterized from the spatiotemporal record of the light diffused by iriodin particles along a line $x / h=4$ in the central plane $y=0$. An excerpt of the signal is displayed in Fig. 4(a), corresponding to about $10 \mathrm{~min}$. Thanks to the $z$ to $-z$ symmetry, only half $(z>0)$ of the signal needs to be presented and further processed.

Eight experiments of $1 \mathrm{~h}$ each have been recorded at a rate of 5 lines per sec, for $R=300,306,310,314$, 320 , and 330. Information has been compressed by detecting the spanwise location $z_{\max }$ of the maximum of the average intensity $I(z)=(1 / z) \sum_{i=1, i_{\max }} I\left(z_{i}, t\right)$, where $i \in[1,256]$ is the index of the pixel in the line corresponding to a given instant of time $t$. The temporal signal $z_{\max }(t)$ is then processed in order to detect the laminar-turbulent alternation. The mean lifetime of laminar and turbulent events $\left\langle\tau_{\text {lam }}\right\rangle$ and $\left\langle\tau_{\text {turb }}\right\rangle$ are then computed, as well as the fraction of time spent in the turbulent state $F_{t}$, called the turbulent fraction. For $R=300$ and 330 less than 20 events were observed; the flow is still too close to the two thresholds $R_{1}^{\prime}$ and $R_{2}^{\prime}$. For $R=306,310,314$, and 320 the number of events varies between 40 and 100 .

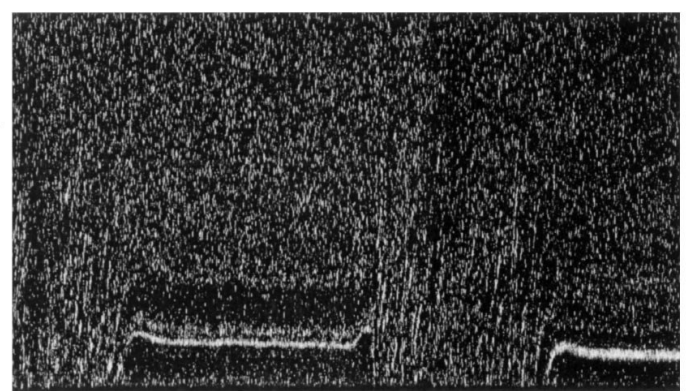

(a)

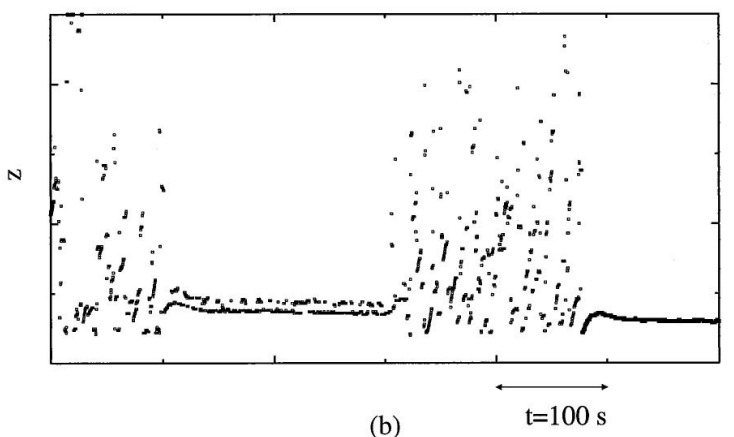

FIG. 4. (a) Excerpt from a spatiotemporal diagram (see text for detail). (b) The corresponding time series $z(t)$.
Figure 5 displays the evolution of these quantities with the Reynolds number. The error bars indicate the measurement accuracy for $R$, as well as for the turbulent fraction, whereas it represents the standard deviation for the laminar and turbulent mean lifetime distribution. The large value of the standard deviation is due to the low number of events at $R=300,330$ and also reveals the proximity of the thresholds $R_{1}^{\prime}$ and $R_{2}^{\prime}$. For the other Reynolds numbers, it is more representative of the width of the distribution since there are enough samples to study the statistics. A detailed study of the distribution is in progress, but we can already mention that two kinds of events can be distinguished: long and short ones. As expected, $F_{t}$ increases with $R$, and its rapid variation allows a good determination of the intermittency threshold $R_{1}^{\prime}$ slightly below 300 .
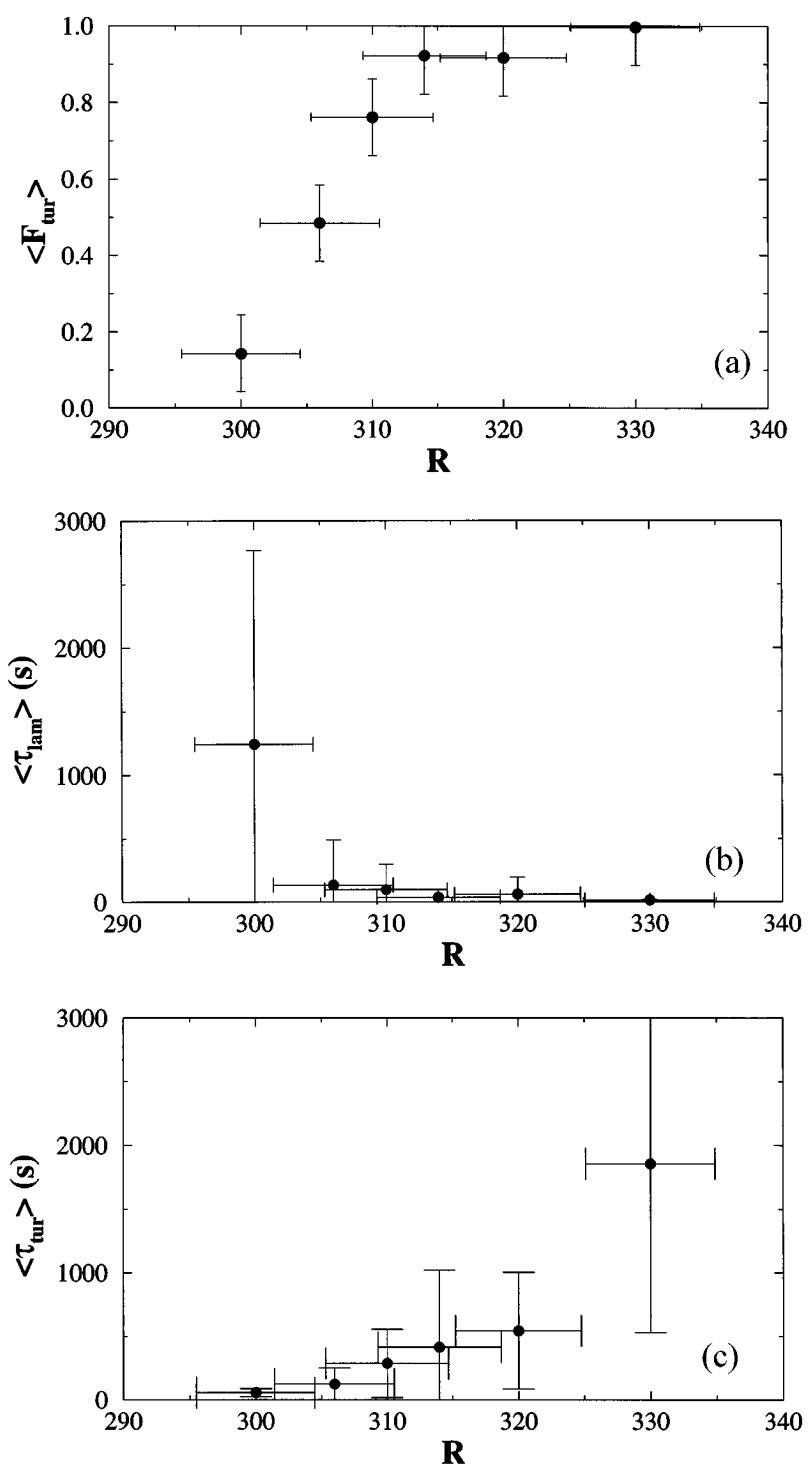

FIG. 5. Evolution of the turbulent fraction (a), the mean lifetime of laminar (b), and turbulent (c) events, with the Reynolds number. 
$\left\langle\tau_{\text {lam }}\right\rangle$ increases rapidly while $\left\langle\tau_{\text {turb }}\right\rangle$ decreases to the finite value $\simeq 240 \mathrm{sec}$ upon decreasing $R$ to $R_{1}^{\prime}$. When increasing $R, F_{t}$ saturates to its maximum value $F_{t}=1$ for $R_{2}^{\prime}=325$ that we recognize as the value beyond which turbulence around the wire becomes sustained.

The destabilization of the streaks around the bead, analyzed here as a temporal process, thus shares strong similarities with the spatiotemporal behavior observed around wires of sufficiently large diameter (see Fig. 2). Whereas turbulent bursts were nucleated at random places along the wire, they are now attached to the bead. In some sense, the structure is spatially frozen and spatiotemporal intermittency is converted into temporal intermittency, which suggests to interpret phenomena within the theory of lowdimensional dynamical systems. In this framework, several routes to chaos have been recognized [1]; however features characteristic of known scenarios (e.g., [13-15]) have not been clearly identified up to now. Some enlightening is expected from the detailed study of the streak breakdown and relaminarization steps that is currently underway [16]. Interestingly, the same kind of process has been observed by Coughlin [17] in numerical simulations of the PCF in confined geometry and can be understood in terms of homoclinic bifurcation of the kind present in simple models such as that derived by Waleffe [18]. The dynamics observed around the bead can then be interpreted as the result of a spatial freezing of the complex spatiotemporal dynamics observed along the wire resulting from such a mechanism. The obtained regime of temporal intermittency, typical of low-dimensional systems, in fact develops in an extended system. As such it keeps a trace of the large number of degrees of freedom that can be easily excited in the spatiotemporal regime. This observation explains why it has been empirically difficult to make a connection with standard scenarios but also suggests implications for the study of the transition to turbulence in other weakly confined systems.
We thank P. Manneville for fruitful discussions and helpful remarks, G. Rousseau and J.F. Pinton for their help in data treatment, and C. Gasquet for technical assistance.

[1] H. G. Schuster, Deterministic Chaos (VCH, Weinham, 1988), 2nd ed.

[2] M. C. Cross and P.C. Hohenberg, Rev. Mod. Phys. 65, 851 (1993).

[3] V. A. Romanov, Funk. An. i Prol. 7, 137 (1970).

[4] H. Reichardt, Z. Angew Math. Mech. Sonderheft 36, 26 (1956).

[5] O. Dauchot and P. Manneville, J. Phys. II (France) 7, 371 (1997).

[6] F. Daviaud, J. Hegseth, and P. Bergé, Phys. Rev. Lett. 69, 2511 (1992).

[7] N. Tillmark and P.H. Alfredsson, J. Fluid Mech. 235, 89 (1992).

[8] O. Dauchot and F. Daviaud, Phys. Fluids 7, 335 (1995).

[9] S. Bottin, O. Dauchot, F. Daviaud, and P. Manneville (to be published).

[10] M. Nagata, J. Fluid Mech. 217, 519 (1990); R. M. Clever and F. H. Busse, J. Fluid Mech. 234, 511 (1992).

[11] O. Dauchot and F. Daviaud, Phys. Fluids 7, 901 (1995).

[12] H. Chaté and P. Manneville, Turbulence: A Tentative Dictionary, edited by P. Tabeling and O. Cardoso (Plenum Press, New York, 1995).

[13] Y. Pomeau and P. Manneville, Commun. Math. Phys. 74, 189 (1980).

[14] C. Grebogi, E. Ott, and J. A. Yorke, Physica (Amsterdam) 7D, 181 (1983).

[15] Y. C. Lai, C. Grebogi, J. A. Yorke, and S. C. Venkatarami, Phys. Rev. Lett. 77, 55 (1996); Y. C. Lai and C. Grebogi, Phys. Rev. Lett. 77, 5047 (1996).

[16] G. Antar, S. Bottin, O. Dauchot, F. Daviaud, and P. Manneville (to be published).

[17] K. Coughlin, J. Fluid Mech. (to be published).

[18] F. Waleffe, Phys. Fluids 9, 883 (1997). 